\title{
Der Hippokratische Eid: ein schwieriges Erbe
}

\author{
Hubert Steinke \\ Prof. Dr. med. Dr. phil., Institut für Medizingeschichte, Universität Bern
}

Seit gut einem Jahr wird in der Schweizerischen Ärztezeitung über den Hippokratischen Eid gestritten. Eine Arbeitsgruppe schlug einen neuen verpflichtenden Eid vor [1], während die Hippokratische Gesellschaft Schweiz die unveränderte Aktualität des Hippokratischen Eides behauptete [2]. Ein Dutzend befürwortende bis radikal ablehnende Kommentare füllten die Leserbrief-Spalten. Im Zentrum dieser Debatte steht die seit Jahrzehnten diskutierte Frage, ob es überzeitliche ethische Prinzipien gibt und ob diese im Hippokratischen Eid formuliert werden. Diese Diskussion sollte in Kenntnis der historischen Verhältnisse geschehen, die im Folgenden kurz geschildert werden.

\section{Das Corpus Hippocraticum}

Das Einzige, was wir dank den kurzen Hinweisen von Platon und Aristoteles über Hippokrates wissen, ist, dass er auf der Insel Kos im Übergang vom 5. zum 4. Jh.v.Chr. als berühmter Arzt und Lehrer tätig war. Was auch immer darüber hinaus behauptet wird, ist gemäss heutigem Wissensstand reine Hypothese oder Fiktion [3]. Beim sogenannten Corpus Hippocraticum handelt es sich um die zentrale Textsammlung der griechischen Medizin, bestehend aus rund 60 Schriften aus dem 5. Jh.v.Chr. bis 2. Jh.n.Chr. Die Texte wurden anonym überliefert und wohl in der Bibliothek von Alexandria ab dem 3. Jh.v.Chr. der Ordnung halber unter dem Namen dieses vor langer Zeit tätigen Arztes verzeichnet. Die Stilisierung von Hippokrates als medizinische Autorität und als zentraler, wenn nicht alleiniger Autor dieses sehr heterogenen, aus sieben Jahrhunderten stammenden und zuweilen widersprüchlichen Sammelwerks setzte v.a. mit Galen im 2. Jh.n.Chr. ein und dauert in Laienkreisen bis heute an. Die professionelle Geschichtsschreibung hat sich im Verlauf des 20. Jh. von der Vorstellung verabschiedet, dass einzelne Texte Hippokrates zugeschrieben werden können.

\section{Der Eid und seine Rezeption}

Der Eid ist eine dieser 60 Schriften und nicht die einzige, die sich zur ärztlichen Ethik äussert [4-6]. Er wird in keiner anderen Schrift dieses Korpus erwähnt und war somit in der griechischen Antike weitgehend unbekannt. Die heutige Forschung kann die Entstehungszeit nicht genau bestimmen und datiert den Text zumeist ins 4., teilweise aber auch erst ins 1. Jh.v.Chr. Die früheste Erwähnung findet sich bei Scribonius Largus im 1. Jh.n. Chr. Spätestens ab dieser Zeit wurde der Eid wohl vereinzelt geschworen. Im Mittelalter breitete er sich zunehmend - oft in christlich abgewandelter Form - aus und wurde zum festen, wenn auch für die ethische Diskussion nicht zentralen Bestandteil der medizinischen Literatur [7]. Erst im Laufe der Renaissance erhielt der Eid den Status eines klassischen Texts, der jedem Studenten und Arzt bekannt war [8]. Damals und in den folgenden Jahrhunderten wurde beim Promotionseid in aller Regel aber nicht der Hippokratische Eid geschworen, sondern ein Fakultätseid, der sich oft an den alten Eid anlehnte. Eine systematische Untersuchung ergab 1981, dass «der hippokratische Eid wahrscheinlich niemals an einer deutschsprachigen medizinischen Fakultät abgelegt worden ist» [9]. Es erstaunt nicht, dass der Eid heute vor allem in den traditionsliebenden amerikanischen Medical Schools verbreitet ist. Gemäss einer Umfrage wurde 1989 an rund der Hälfte der amerikanischen Universitäten "eine Version" des Hippokratischen Eides geschworen [10].

\section{Offene Interpretationen}

Da der Text kurz und der Entstehungszusammenhang unbekannt ist, muss die Übersetzung und Interpretation an mehreren Stellen offenbleiben. Umstritten sind insbesondere diejenigen Teile, die nicht im Einklang mit den übrigen Texten des Corpus Hippocraticum stehen. Während etwa das Prinzip des Nutzens und Nicht-Schadens auch in anderen Traktaten anzutreffen ist, zeugt die Bestimmung, «nicht zu schneiden, jedenfalls Steinleidende nicht», von einer Distanzierung von der Chirurgie, wie sie in keiner anderen Quelle zu finden ist. Um den Widerspruch zum Corpus aufzulösen, haben Philologen versucht, die Stelle so zu deuten, dass ausschliesslich der Steinschnitt gemeint 


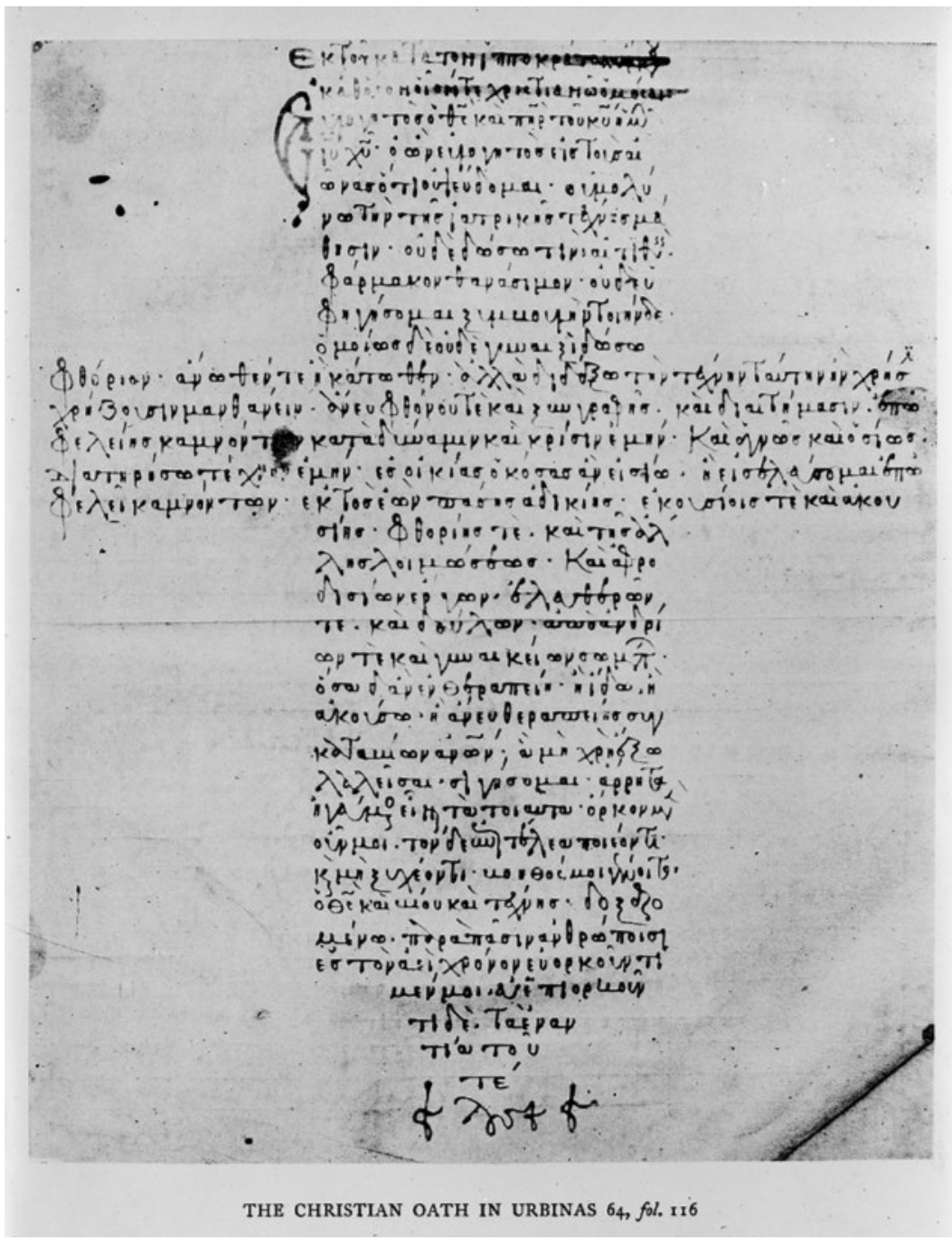

Biblioteca Apostolica Vaticana, Codex Urbinas Graecus 64, fol. 116. Die Handschrift aus dem 12. Jahrhundert zeigt den Eid in Kreuzform und mit einem an christliche Vorstellungen angepassten Text. (C) Wellcome Library, London ist. Noch schwieriger ist der Passus zur Abtreibung, der oft als grundsätzliches Abtreibungsverbot interpretiert wird, aber auch nur die Rückweisung der bestimmten Form des Aborts mit einem Tampon bedeuten kann. Diese letztere Lesung wäre besser mit der Medizin der klassischen Zeit vereinbar, in der Kindesaussetzung, Kindstötung und vielfältige Formen der Abtreibung ohne gesellschaftliche Ächtung belegt sind [6]. Ähnlich verhält es sich mit der Stelle zur Giftabgabe, die sowohl als Verbot der Beihilfe zum Suizid wie auch als Zurückweisung der Beteiligung an einem Giftmord (etwa als Leibarzt eines Herrschers) verstanden werden kann. Letztere Interpretation erscheint deswegen plausibel, weil in der antiken Literatur kein Fall von ärztlich assistiertem Suizid durch Gift beschrieben ist, wohl aber die verbreitete Angst vor Gift- mord [11]. Die ursprüngliche, vom Autor gemeinte Bedeutung dieser Stellen muss also unklar bleiben, auch wenn sie in späterer, christlicher Zeit als allgemeines Verbot der Beihilfe zu Abort und Suizid interpretiert wurden.

\section{In der Antike unbekannt}

Ganz ungewöhnlich für die griechische Antike ist die Verpflichtung, für den Lehrer zu sorgen, sowie der stark religiöse Ton mit der Anrufung der Götter und dem Versprechen, das ganze Leben in Reinheit zu führen. Diese weit über die Ausbildung und berufliche Praxis hinausgehenden Pflichten unterscheiden sich stark von den viel pragmatischeren, auf vertrauenswürdiges Auftreten und zielführende ärztliche Praxis konzentrierten Verhaltensregeln im übrigen Corpus Hippocraticum [12]. Sie deuten darauf hin, dass der Eid nur für eine kleinere Gemeinschaft von Bedeutung gewesen ist und daher kaum bekannt war. Dies ist insofern einleuchtend, als es zu dieser Zeit keine einheitliche Ärzteschaft gab, sondern eine Vielfalt konkurrierender unterschiedlicher Heiler.

\section{Vereinnahmungen}

Idealisierende Rückbesinnungen auf die "guten alten Zeiten" finden vor allem in Phasen der Unsicherheit statt. So war es auch in der "Krise der Medizin» in den 1920er und 1930er Jahren oder in der NS-Zeit. Durch selektive Wahrnehmung und ideologisch fundierte Vereinnahmung konstruierte man das Zerrbild einer ununterbrochenen Tradition ärztlicher Moral, etwa in der 1942 erschienenen, mit einem Vorwort von Heinrich Himmler eingeleiteten Sammlung Hippokratischer Ethik unter dem Titel Ewiges Arzttum [13]. Solche Publikationen sagen wenig über die antike Medizin, aber viel über die Gesinnung der Herausgeber aus [14]. Auch heute lässt sich die ungebrochene Gültigkeit des Hippokratischen Eids nur durch Leugnung der Interpretationsprobleme und Ausblendung einzelner Teile behaupten. Es ist schwer nachvollziehbar, wie man etwa die Bestimmung, medizinisches Wissen nur mit den Schülern, sonst aber mit niemandem zu teilen, heute verteidigen will. Dieses Prinzip machte nur Sinn für eine Gruppe, die aussenstehenden Konkurrenten aus Berufsinteressen den Zugang zu exklusivem Wissen verwehren wollte. Wer sich trotz der offensichtlichen radikalen Veränderungen der beruflichen und gesellschaftlichen Verhältnisse auf eine angeblich ungebrochene Tradition beruft und damit beansprucht, über historisches Bewusstsein zu verfügen, zeigt, dass ihm eben gerade dieses abgeht. 


\section{Der Eid als Symbol}

Der Hippokratische Eid hat den wichtigsten Test nicht bestanden. Im Nürnberger Ärzteprozess beriefen sich sowohl Anklage wie Verteidigung auf ihn, in der Urteilsbegründung wurde er aber nicht mehr erwähnt. Es hatte sich gezeigt, dass der Eid keine Formel lieferte, um das ethische Verhalten des modernen Arztes zu beurteilen [15]. Im Anschluss an den Prozess wurde daher das Genfer Gelöbnis und der Nürnberger Kodex (1948) zur Regelung medizinischer Versuche am Menschen verfasst. Aber weder das Genfer Gelöbnis noch ein neu formulierter Eid kann die Komplexität der heutigen Medizin abbilden. Er kann ausführliche ethische Richtlinien, wie sie die SAMW erarbeitet, nicht ersetzen. Ein neuer Eid würde wohl - wie der Hippokratische Eid weniger als präzis formulierter Text wirken, sondern vor allem als Symbol, das durchaus seinen Sinn haben kann. Man muss sich allerdings bewusst sein, dass auch ein neuer Eid nicht gegen die Gefahren der Selbststilisierung, Vereinnahmung und Ausgrenzung der nicht Vereidigten gefeit ist. Dennoch kann es sich lohnen, in Kenntnis der Problematik des antiken Eids und

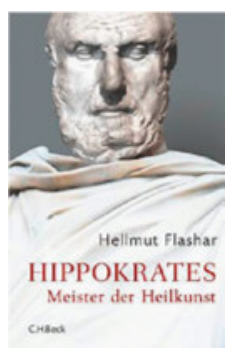

Hellmut Flashar Hippokrates.

Meister der Heilkunst. Leben und Werk

München: C.H.Beck; 2016. 26.95 EUR. 297 Seiten. ISBN 978-3-406-69746-3

\section{Gelungenes Buch, misslungene Werbung}

Vor wenigen Wochen ist ein neues Buch über den Namensgeber des Hippokratischen Eids erschienen. Der Autor ist ein bekannter Altertumswissenschafter, der die Eigenart der hippokratischen Medizin aus der Sicht des Philologen einem breiteren Publikum bekannt machen möchte. Er bringt uns die bemerkenswerte Vielgestaltigkeit der antiken Medizin näher und lässt uns damit in eine vergangene Epoche eintauchen, macht aber gleichzeitig auch die Verständnisschwierigkeiten deutlich, die der heutige Leser hat. Nicht zuletzt weist er auf die schon früh einsetzende Idealisierung und Vereinnahmung der historisch nur sehr schwach dokumentierten Person hin. Erstaunlich, wie die Differenzierung, die das Buch prägt, im Klappentext völlig über Bord geworfen wird. Dort ist zu lesen: «Bis auf den heutigen Tag ist der hippokratische Eid der Maßstab ethischen Handelns in der abendländisch geprägten Medizin.» Es scheint, dass sich mit der alten Behauptung der überzeitlichen hippokratischen Ethik heute immer noch am besten hausieren lässt. seiner Rezeption darüber zu diskutieren. Den Hippokratischen Eid aber in seinem originalen Wortlaut heute noch als gültige ethische Richtlinie zu benutzen, erscheint vor dem Hintergrund der geschilderten historischen Verhältnisse als geradezu absurd.

\section{Literatur}

1 Giger M. Ein Eid für heutige Ärztinnen und Ärzte. Schweiz. Ärztezeitung 2015;96(25):930-4.

2 Mattli J, Knirsch U, Kiesse R, Vuilleumier-Koch S. Zur Bedeutung des Hippokratischen Eides in der heutigen Zeit. Schweiz. Ärztezeitung 2016;97(23):854-6.

3 Leven KH. Die Erfindung des Hippokrates - Eid, Roman und Corpus Hippocraticum. In: Tröhler U, Reiter-Theil S (Hg.) Ethik und Medizin 1947-1997. Was leistet die Kodifizierung von Ethik? Göttingen 1997:19-40.

4 Grundlegend, wenn auch heute teilweise überholt ist Lichtenthaeler C: Der Eid des Hippokrates. Ursprung und Bedeutung. Köln 1984.

5 Eine gute Einführung liefert Boschung U. Der hippokratische Eid Überlieferung, Wirkungsgeschichte und medizinhistorische Interpretation. In: Ausfeld-Hafter B (Hg.). Der hippokratische Eid und die heutige Medizin. Bern 2003:9-26.

6 Für eine neuere Übersicht und Bibliographie vgl. Schubert C. Der hippokratische Eid. Medizin und Ethik von der Antike bis heute. Darmstadt 2005.

7 Galvão-Sobrinho C. Hippocratic Ideals, Medical Ethics, and the Practice of Medicine in the Early Middle Ages: The Legacy of the Hippocratic Oath. Journal of the History of Medicine and Allied Sciences 1996(51):438-55.

8 Rütten T. Receptions of the Hippocratic Oath in the Renaissance: The Prohibition of Abortion as a Case Study in Reception. Journal of the History of Medicine and Allied Sciences 1996(51):456-83.

9 Nolte W. Der hippokratische Eid und die Abschlusseide der früheren und jetzigen deutschsprachigen Hochschulen. Diss. med. Bochum 1981, 91.

10 Smith DC. The Hippocratic Oath and Modern Medicine. Journal of the History of Medicine and Allied Sciences 1996(51):484-500.

11 Rütten T. Medizinethische Themen in den deontologischen Schriften des "Corpus Hippocraticum». In: Flashar H, Jouanna J (Hg.). Médecine et morale dans l'antiquité. Genève 1997:65-120.

12 Nutton V. Beyond the Hippocratic Oath. In: Wear A, Geyer-Kordesch J, French R (Hg.). Doctors and Ethics. The Earlier Historical Setting of Professional Ethics. Amsterdam 1993:10-37.

13 Frewer A, Bruns F. «Ewiges Arzttum» oder «neue Medizinethik» 1939-1945? Hippokrates und Historiker im Dienst des Krieges. Medizinhistorisches Journal 2003(38):313-36.

14 Vgl. allgemein dazu Bruchhausen W. Abschied von Hippokrates? Historische Argumentationen in der bioethischen Kritik am ärztlichen Ethos. In: Bruchhausen W, Hofer HG (Hg.). Ärztliches Ethos im Kontext. Historische, phänomenologische und didaktische Analysen. Göttingen 2010:75-98.

15 Leven KH. Hippokrates im 20. Jahrhundert: Ärztliches Selbstbild, Idealbild und Zerrbild. In: Leven KH, Prüll CR (Hg.). Selbstbilder des Arztes im 20. Jahrhundert. Medizinhistorische und medizinethische Aspekte. Freiburg i.Br. 1994:39-96. 